\title{
Preparation and evaluation of prednisolone sodium phosphate ocusert for controlled drug delivery
}

\author{
Karan Sharma, Navneet Nagpal \\ Department of Pharmaceutics, Faculty of Medical, Paramedical, Pharmaceutical and Health Sciences, Khalsa University, Amritsar, Punjab, India
}

\begin{abstract}
The intent of research was to formulate and evaluate controlled release drug delivery system of ocusert of prednisolone sodium phosphate, corticosteroid for the treatment of viral conjunctivitis. Ocusert are sterile preparation having drug as dispersion or as solution in the polymeric base. Ocusert were formulated using different polymers such as hydroxypropyl methyl cellulose E-15, ethyl cellulose, and Eudragit RL-100 at various concentrations and combinations. Films were prepared by mercury casting method using different ratios of polymers. Selected physiochemical properties such as thickness, weight, percentage moisture absorption, and in-vitro release pattern of Prednisolone sodium phosphate ocusert were studied and reported.
\end{abstract}

Correspondence:

Dr. Navneet Nagpal,

Khalsa College of Pharmacy, GT Road,

Amritsar - 143 002, Punjab, India.

E-mail: n.nagpal721@gmail.com
Keywords: Hydroxypropyl methyl cellulose E-15, ocusert, prednisolone sodium phosphate

\section{Introduction}

Ophthalmic preparations are sterile products free from foreign particles. The conventional ophthalmic preparation includes drops, solutions, suspension, and ointments. Most frequently used ophthalmic preparations are solutions but these conventional preparations having various drawbacks such as shorter duration of action reduced corneal contact time leading to poor bioavailability, frequent administration, and patient non-compliance. In the present study, ocusert of prednisolone sodium phosphate were prepared to overcome the disadvantages of conventional ophthalmic and to achieve better patient compliance. ${ }^{[1]}$ Ocuserts or ophthalmic inserts defined as sterile preparations with solid or semi-solid consistency, whose size and shape are especially designed for ophthalmic application. They are essentially composed of polymeric support containing drug, the latter being incorporated as dispersion or a solution in a polymeric support. The advantages of ocuserts are increase ocular residence, prolonged drug activity, higher bioavailability with respect to standard vehicles, release of drug at a slow, constant rate, accurate dosing. The reduction of systemic absorption of drug has better patient compliance due to reduced frequency of administration and fewer incidences of visual and systemic side effects. ${ }^{[2,3]}$

\section{Access this article online}

Website: http://www.jpbs-online.com

E-ISSN: 2321-0125

DOI: 10.31555/jpbs/2018/6/1/5-8

How to cite this article: Sharma K, Nagpal N. Preparation and evaluation of prednisolone sodium phosphate ocusert for controlled drug delivery. J Pharm BioSci 2018;6(1):5-8.

Source of Support: Nil, Conflict of Interest: None declared.
The objective of this study is to formulate a small, liable flat disk, oval or round with a desired thickness for the treatment of viral conjunctivitis. The main purpose of preparing ocusert is to increase ocular bioavailability by increasing the corneal contact time. Fewer administrations provide patient compliance.

Viral conjunctivitis or pink eye is a common, self-limiting condition that is typically caused by adenovirus. ${ }^{[4]}$

Prednisolone sodium phosphate is corticosteroid drug effective in the treatment of viral conjunctivitis. This drug decreases inflammation by suppressing migration of polymorphonuclear leukocytes and reversing increasing capillary permeability. Hence, an attempt has been made in the present work to make an ocular film with the polymers hydroxypropyl methyl cellulose (HPMC), ethyl cellulose (EC) and Eudragit RL100. Ocular films of prednisolone sodium phosphate were prepared by mercury casting method..$^{[4]}$

\section{Materials and Methods}

Prednisolone sodium phosphate was procured from Ananta Pharmaceutical Pvt., Ltd., Sri Ganganagar (Rajasthan). EC, HPMC, Eudragit RL-100 were purchased from Sigma Aldrich. polyethylene glycol 400 (PEG-400) and chloroform, hydrochloric acid were purchased from Merk. All chemicals were of analytical grade.

\section{Preparation of ocuserts}

\section{Preparation of drug reservoir membrane}

Mercury casting method is used for preparation of ocusert. The calculated quantity of prednisolone sodium phosphate was accurately weighed 
and dissolved in distilled water. Polymer was weighed and dissolved in distilled water separately in another beaker. Then clear drug solution was poured into polymer solution with constant stirring to get a homogeneous solution. The required amount of PEG-400 was added and mixed well. The resulting solution was casted over the mercury surface. ${ }^{[5]}$

\section{Preparation of rate controlling membrane}

EC was dissolved in chloroform. Required quantity of PEG-400 was added and stirred until to get a clear solution. Then, it was poured over a clear glass plate and allowed to dry. ${ }^{[6]}$

\section{Sealing}

The prepared rate controlling membrane and drug reservoir were cut into circular shape using a special mold after sufficient drying. The drug reservoir was sealed on both sides using EC having 8 and $10 \mathrm{~mm}$ diameter, respectively. ${ }^{[7]}$ The proportions of HPMC, EC, Eudragit, and plasticizers were shown in Table 1.

\section{Calculation:}

Amount of drug present in one ocusert $=1.5 \mathrm{mg}$ of prednisolone sodium phosphate.

Diameter of the proposed ocusert $=0.8 \mathrm{~cm}$.

Therefore area of the proposed ocusert $=0.503 \mathrm{~cm}^{2}$.

Diameter of the ring $=4 \mathrm{~cm}$.

Radius of the ring $=2 \mathrm{~cm}$.

Area of the ring $=12.56 \mathrm{~cm}^{2}$.

Number of ocusert present in the proposed area of the ring $=12.56 / 0.503=25$ ocusert.

Amount of drug present in 1 ocusert $=1.5 \mathrm{mg}$.

Amount of drug present in 25 ocuserts $=37.5 \mathrm{mg}$.

\section{Evaluation of ocuserts}

\section{Thickness}

Thickness was measured using a screw gauge at different places of the ocusert and the average was calculated..$^{[5]}$

\section{Weight}

Weight was calculated on digital balance. Three ocuserts were weighed individually and the average weight was calculated. ${ }^{[6]}$

\section{Drug content}

Three ocuserts were taken and cut into small pieces, put into $100 \mathrm{ml}$ buffer ( $\mathrm{pH}$ 7.4) and shaken continuously until they dissolve. The solution was ultra sonicated for $15 \mathrm{~min}$. After filtration, the drug was suitably diluted and analyzed at $246 \mathrm{~nm}$ in ultraviolet (UV) visible spectrophotometer. ${ }^{[6]}$

\section{Folding endurance}

Folding endurance was determined by repeatedly folding the film at the same place till breaking or appearance of breaking signs. The number of times the film could be folded at the same place without breaking gives the folding endurance value. ${ }^{[7]}$

\section{Moisture uptake}

The ocuserts were subjected to desiccation over calcium chloride at room temperature for $48 \mathrm{~h}$. These ocuserts were then weighed and the weight was recorded as initial weight. The ocuserts were then exposed to $75 \%$ relative humidity (a saturated solution of ammonium chloride) in a desiccator until a constant weight of the ocuserts was obtained. The percentage of moisture uptake was calculated as the difference between final and initial weight with respect to initial weight. ${ }^{[8]}$

\section{Surface $\mathbf{p H}$}

The ocuserts were first allowed to swell by keeping them in contact with $5 \mathrm{ml}$ of distilled water for $1 \mathrm{~h}$ in petri dish. $\mathrm{pH}$ was noted by bringing the glass electrode near the surface of the formulation (ocusert) and allowing it to equilibrate for $1 \mathrm{~min} .{ }^{[8]}$

\section{In vitro release studies}

In vitro release studies were carried out using bichambered donor receiver compartment model (Franz diffusion cell). The diffusion cell membrane (prehydrated cellophane) was tied to one end of the open cylinder, which acted as donor compartment. The ocular insert was placed on a dialysis membrane, which was in contact with receptor medium comprising $40 \mathrm{ml}$ of simulated tear fluid (STF) $(\mathrm{pH}=7.4)$. The content of the receptor compartment was stirred continuously using a magnetic stirrer and temperature was maintained at $37^{\circ} \mathrm{C} \pm$ $0.50^{\circ} \mathrm{C}$. The receptor medium was stirred continuously at $20 \mathrm{rpm}$ to simulate blinking action of eyelids. At specific time interval, $1 \mathrm{ml}$ aliquot of the solution was withdrawn and replaced with fresh STF and required dilutions were made. The aliquot was analyzed for drug content was analyzed using UV spectrophotometer at $246 \mathrm{~nm}$ against reference standard using STF as blank. ${ }^{[9,10]}$

Table 1: Composition of ocuserts containing prednisolone sodium phosphate

\begin{tabular}{|c|c|c|c|c|c|c|c|c|}
\hline \multirow{2}{*}{$\begin{array}{l}\text { Formulation } \\
\text { code }\end{array}$} & \multicolumn{2}{|c|}{ Rate controlling membrane } & \multicolumn{6}{|c|}{ Drug reservoir membrane } \\
\hline & EC $(\%)$ & PEG $400(\mathrm{ml})$ & Drug $(\%)$ & HPMC E-15 (\%) & $\begin{array}{c}\text { Eudragit } \\
\text { RL-100 (\%) }\end{array}$ & $\begin{array}{l}\text { PEG } 400 \\
(\mathrm{ml})\end{array}$ & $\begin{array}{c}\text { Ethanol } \\
(\mathrm{ml})\end{array}$ & $\begin{array}{l}\text { Purified water q.s. } \\
(\mathrm{ml})\end{array}$ \\
\hline F1 & 3 & 0.5 & 1 & 3 & - & 0.5 & 3 & 10 \\
\hline F2 & 4 & 0.5 & 1 & 3 & - & 0.5 & 3 & 10 \\
\hline F3 & 5 & 0.5 & 1 & 3 & - & 0.5 & 3 & 10 \\
\hline $\mathrm{F} 4$ & 3 & 0.5 & 1 & - & 3 & 0.5 & 3 & 10 \\
\hline F5 & 4 & 0.5 & 1 & - & 3 & 0.5 & 3 & 10 \\
\hline F6 & 5 & 0.5 & 1 & & 3 & 0.5 & 3 & 10 \\
\hline
\end{tabular}

HPMC: Hydroxypropyl methyl cellulose, EC: Ethyl cellulose, PEG: Polyethylene glycol 
Table 2: Evaluation parameters of ocusert

\begin{tabular}{|c|c|c|c|c|c|c|}
\hline \multirow{2}{*}{$\begin{array}{l}\text { Formulation } \\
\text { code }\end{array}$} & \multicolumn{5}{|c|}{ Mean \pm SD } & \multirow{2}{*}{ Surface $\mathbf{p H}$} \\
\hline & Thickness (mm) & Weight (mg) & Drug content $(\%)$ & Folding endurance & Moisture uptake & \\
\hline $\mathrm{F} 1$ & $0.33 \pm 0.054$ & $18.58 \pm 1.39$ & $0.621 \pm 0.008$ & $85.2 \pm 2.91$ & $6.39 \pm 0.20$ & 7.3 \\
\hline F2 & $0.45 \pm 0.062$ & $20.34 \pm 1.77$ & $0.578 \pm 0.005$ & $78.6 \pm 1.73$ & $7.45 \pm 0.15$ & 7.8 \\
\hline F3 & $0.21 \pm 0.043$ & $16.21 \pm 1.63$ & $0.456 \pm 0.005$ & $76.4 \pm 1.23$ & $9.23 \pm 0.12$ & 7.1 \\
\hline $\mathrm{F} 4$ & $0.127 \pm 0.069$ & $14.23 \pm 1.32$ & $0.432 \pm 0.006$ & $82.5 \pm 2.55$ & $8.34 \pm 0.07$ & 6.7 \\
\hline F5 & $0.106 \pm 0.093$ & $12.45 \pm 1.44$ & $0.411 \pm 0.007$ & $79.3 \pm 3.22$ & $7.12 \pm 0.09$ & 6.2 \\
\hline F6 & $0.182 \pm 0.056$ & $15.56 \pm 1.48$ & $0.401 \pm 0.004$ & $80.9 \pm 2.01$ & $5.86 \pm 0.14$ & 6.6 \\
\hline
\end{tabular}

SD: Standard deviation

To understand the mechanism and kinetics of drug release, the results of in vitro drug release study were fitted with various kinetic equations such as zero order, first order, and Higuchi matrix. Based on the " $R$ " value, the best-fit model was selected.

\section{Results and Discussion}

\section{Uniformity of thickness}

The prepared films were evaluated for the thickness of each film using a Vernier caliper scale and the average thickness of the fine ocusert was recorded and shown in Table 2. The main thickness, standard deviation, and percent coefficient of variation were calculated. All the six formulation measured thickness with low standard deviation values ensured the uniformity of the films prepared by mercury casting method.

\section{Uniformity of weight}

Films were weighted and the weights of the inserts were found to be in the range of $20.34 \pm 1.39$ and $15.56 \pm 1.32$.

\section{Drug content}

The drug content of all the films was found to be in the range of 0.621 \pm 0.005 and $0.432 \pm 0.004$. The result indicates that various results were found using different polymers.

\section{Percentage moisture absorption}

Percentage moisture absorption was calculated for all six films in triplicate. The moisture absorption was more in the formulation where hydrophilic polymers were present. In formulation 3, the result obtained was $9.23 \pm 0.12$, as the film contains HPMC as polymer. In formulation 6 , the results were found minimum percentage moisture absorption maybe because of hydrophobic nature of polymer.

\section{Folding endurance}

It was done manually by folding the film repeatedly at a point till it broke. Folding endurance was found to be the highest for F1 and lowest in F3.

\section{Surface $\mathrm{pH}$}

Surface $\mathrm{pH}$ for all the six films was within the range of 7.8-7.1 and 6.76.2. In general, the $\mathrm{pH}$ of ophthalmic formulation should be within 4.5-11.5 so it was found to be good result for all six formulations.

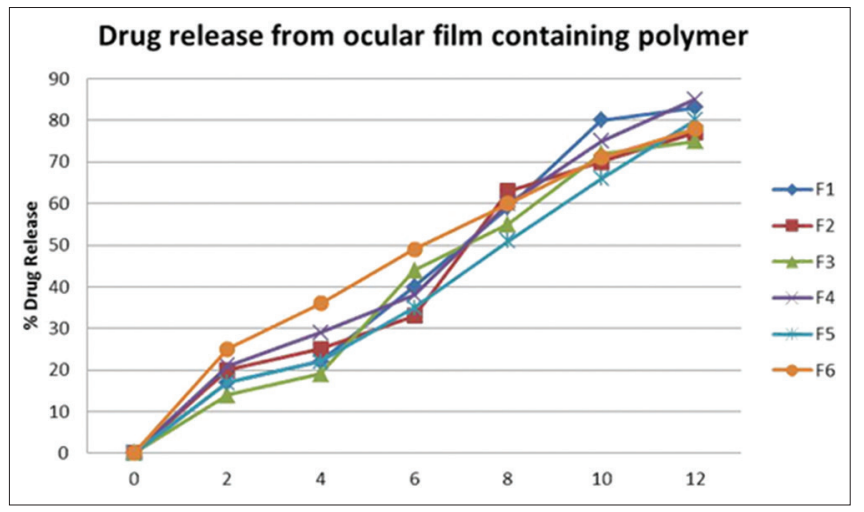

Figure 1: In-vitro drug release of the drug reservoir

\section{In vitro release studies}

All the six have been able to release the drug above $77 \%$ in $12 \mathrm{~h}$. Among these formulations, F1 and F4 showed higher drug release as compare to others. In both these formulations, we used 3\% of EC in rate controlling membrane. In contrast, F2, F3, F5, and F6 showed lesser drug release because of high percentage of EC in rate controlling membrane. F1 releases $83 \%$ in $12 \mathrm{~h}$ and F4 releases $85 \%$ in $12 \mathrm{~h}$ (Figure 1).

\section{Conclusion}

The research work was to formulate and evaluate ocusert of prednisolone sodium phosphate using various polymers such as HPMC, EUDRAGIT, and EC for control release. Prednisolone sodium phosphate is corticosteroid drug effective in the treatment of viral conjunctivitis. The various evaluations and the stability studies were done to prove ocuserts are stable. Finally, it could be concluded from all the studies conducted that the formulation was a viable alternative to the conventional eye drops by virtue of its ability to enhance bioavailability through controlled drug delivery, longer precorneal residence time, ease and reduced frequency of administration resulting in better patient compliance.

\section{References}

1. Gan L, Wang J, Jiang M, Bartlett H, Ouyang D, Eperjesi F, et al. Recent advances in topical ophthalmic drug delivery with lipid-based nanocarriers. Drug Discov Today 2013;18:290-7.

2. Tangri P, Khurana S. Basics of ocular drug delivery systems. Int J Res Pharm Biomed Sci 2011;2:1541-52. 
3. Bourlais CL, Acar L, Zia H, Sado PA, Needham T, Leverge R. Ophthalmic drug delivery systems - Recent advances. Prog Retin Eye Res 1998;17:33-58.

4. Kuo SC, Shen SC, Chang SW, Huang SC, Hsiao CH. Corneal superinfection in acute viral conjunctivitis in young children. J Pediatr Ophthalmol Strabismus 2008;45:374-6

5. TanwarYS, Patel D, Sisadia SS. In-vitro and in-vivo evaluation of ocular inserts of ofloxacin. DARU 2007;15:139-45.

6. Patel UL, Chotai NP, Nagda CD. Design and evaluation of ocular drug delivery system for controlled delivery of gatifloxacin sesquehydrate: In vitro and in vivo evaluation. Pharm Dev Technol 2012;17:15-22.
7. Kamath UR, Singh UV, Udupa N. Evaluation of ciprofloxacin hydrochloride ocular preparations. Indian J Pharm Sci 1993;55:148-50.

8. Sankar V, Chandrasekaran AK, Durga S, Geetha G, Ravichandran V, Vijayakumar A, et al.Design and evaluation of diclofenac sodium ophthalmic inserts. Acta Pharm Sci 2006;48:5-10.

9. Charoo NA, Kohli K, Ali A, Anwer A. Ophthalmic delivery of ciprofloxacin hydrochloride from different polymer formulations: In vitro and in vivo studies. Drug Dev Ind Pharm 2003;29:215-21.

10. Rastogi SK, Vayas N, Mishra B. "In-vitro and in-vivo evaluation of pilocarpine hydrochloride ocuserts,” The Eastern Pharmacist 1996: 99: 41-43. 